\title{
Saddle Pulmonary Embolism and Thrombus-in-Transit Straddling the Patent Foramen Ovale 28 Days After COVID Symptom Onset
}

\author{
Kana Fujikura ${ }^{1}$, Joao Fontes ${ }^{2}$, and Cynthia Taub² \\ ${ }^{1}$ Montefiore Hospital and Medical Center \\ ${ }^{2}$ Montefiore Medical Center
}

June 1, 2020

\begin{abstract}
We present a late presentation of saddle pulmonary embolism and thrombus-in-transit straddle the patent foramen on patient who successfully recovered from severe acute respiratory syndrome coronavirus-2 (COVID-19) pneumonia. Seven days postdischarge (i.e. 28 days after initial COVID-19 symptom onset), she was readmitted to hospital for severe dyspnea. Computer tomography angiogram and echocardiography confirmed the diagnosis. Severe pro-inflammatory and pro-thrombotic states with endothelial involvement have been reported associated with severe COVID-19 infection. However the duration of hypercoagulable state has not yet known. This case highlights the risk of thromboembolic phenomena for prolonged periods of times after recovering from COVID-19 pneumonia.
\end{abstract}

\section{Keywords:}

2D echocardiography, computed tomography, right atrial thrombus, pulmonary artery, embolism

\section{INTRODUCTION}

The COVID-19 infection is now confirmed approximately 6 million worldwide, and over 1.7 million in the United States (1). Acute thrombotic events can occur during admission of COVID-19 (2). Among patients with severe COVID-19 symptom admitted to ICU, high incidence of venous thromboembolism has been reported despite prophylaxis (3). Patients who developed acute respiratory distress syndrome (ARDS) associated with COVID-19 showed significantly higher incidence of pulmonary embolism compared to ARDS without COVID-19 (4). However, data of hypercoagulability is still not available post-discharge after recovering from COVID-19 ARVD (5).

\section{CASE PRESENTATION}

77-year-old female with prior medical history includes stroke, diabetes mellitus, hypertension, and breast cancer in remission presented with severe dyspnea. She was initially admitted for severe coronavirus disease-2019 (COVID-19) pneumonia. At index admission, laboratory studies showed normal leucocyte count, elevated lactic dehydrogenase $(804 \mathrm{U} / \mathrm{L})$, D-dimer $(>20.00 \mu \mathrm{g} / \mathrm{mL})$ and C-reactive protein $(11.7 \mathrm{mg} / \mathrm{dL})$. Echocardiography showed right ventricular enlargement and hypokinesia with estimated pulmonary artery systolic pressure (PASP) of $37 \mathrm{mmHg}$. Treatment included hydroxychloroquine, azithromycin, vancomycin, oxygen, and full-dose unfractionated heparin. She recovered, and was discharged after 17 days of hospitalization. Upon discharge, heparin was transitioned to apixaban, however she did not fill a prescription.

Seven days after hospital discharge, she presented again for severe dyspnea. She was tachycardiac at 140 bpm, normotensive, hypoxic with peripheral capillary oxygen saturation of $85 \%$ on room air. Repeat Ddimer was $9.34 \mu \mathrm{g} / \mathrm{mL}$, higher than that of a week prior $(3.67 \mu \mathrm{g} / \mathrm{mL})$. Pro-B natriuretic peptide was normal. Electrocardiogram showed sinus tachycardia and bilateral atrial enlargement (Figure 1). Computed 
tomography angiogram revealed a large saddle pulmonary embolism (Figure 2). Echocardiography showed the right ventricular dilatation and hypokinesia with classic McConnel's sign (Figure 3A, Video 1). A mobile sausage-like mass was observed in the right atrium, straggled the patent foramen ovale, which was consistent with thrombus-in-transit. Color Doppler showed moderate tricuspid regurgitation (Figure 3B, Video 2). PASP was estimated as $62 \mathrm{mmHg}$. Venous duplex Doppler showed acute occlusive thrombosis in gastrocnemius and popliteal veins of the right lower extremity. She had no clinical signs and symptoms for stroke.

Heparin infusion was initiated and hemodynamics were closely monitored in the intensive care unit. Her blood pressure remained stable, therefore tissue plasminogen activator, extracorporeal membrane oxygenation, or thrombectomy were deferred. Tachycardia resolved, and she was discharged home with enoxaparin. Follow up echocardiogram was scheduled as outpatient.

\section{DISCUSSION}

A COVID-19 autopsy study revealed pulmonary embolism as a direct cause of death in one third of cases and found unexpected deep venous thrombosis in over $50 \%$ cases (6). Severe pro-inflammatory and prothrombotic state is a plausible explanation of hypercoagulability associated with COVID-19 infection (7). Endothelial injury is also postulated as a potential cause for in situ thrombosis (8). It is not yet well known how long this hypercoagulable state persists after recovery from COVID-19 infection.

Our patient failed to continue anticoagulation after hospital discharge and developed acute pulmonary embolism with coexistence of thrombus-in-transit 28 days after initial COVID-19 symptom onset. These floating thrombi are in transit from the legs to the pulmonary arteries and hence can embolize at any time, thus necessitating emergency treatment (9). Due to its transient nature, thrombus-in-transit is a rare finding. When the foramen ovale is patent, the floating thrombi may extend through the 'hole' and may cause stroke by paradoxical embolism.

As for port-discharge thromboprophylaxis, there is still not enough data on patients who survived severe COVID-19 infection. At this time, the survivors of severe COVID-19 infection are recommended to follow a general guideline $(5,10)$. This case highlights the risk of thromboembolic phenomena for prolonged periods of times after recovering from acute respiratory distress syndrome associated with COVID-19. More data is needed to determine the optimal duration of anticoagulation treatment.

\section{Figure legends:}

Figure 1. Electrocardiogram (A) 9 days before admission, and (B) at emergency department with tachycardia and biatrial enlargement.

Figure 2. Computed tomography angiogram of (A) axial and (B) coronal slices shows saddle embolism.

Figure 3. Echocardiography of (A) apical 4-chamber view with a sausage-like thrombus in the right atrium straddle the patent foramen ovale which was consistent with thrombus-in-transit, and (B) color Doppler showing tricuspid regurgitation jet hitting the thrombus. Red arrows show thrombus.

Video 1. Echocardiography of apical 4-chamber view showed the right ventricular dilatation and hypokinesia with classic McConnel's sign. There was a sausage-like thrombus in the right atrium straddle the patent foramen ovale which was consistent with thrombus-in-transit.

Video 2. Echocardiography with color Doppler zooming up the right atrium. There was tricuspid regurgitation jet directing toward the thrombus.

\section{References:}

1. Johns Hopkins University and Medicine, Coronavirus Resource Center. COVID-19 Dashboard by the Center for systems Science and Engineering at Johns Hopkins University. 5/29/2020. Available at: https://coronavirus.jhu.edu/map.html. Accessed May 29, 2020. 
2. Wichmann D, Sperhake JP, Lütgehetmann M, et al. Autopsy Findings and Venous Thromboembolism in Patients With COVID-19: A Prospective Cohort Study. Ann Intern Med 2020 May 6 [ahead of print]; https://doi.org/10.7326/M20-2003.

3. Klok FA, Kruip MJHA, van der Meer NJM, et al. Incidence of thrombotic complications in critically ill ICU patients with COVID-19. Thromb Res. 2020 Apr 10:S0049-3848(20)30120-1. [ahead of print]; https://doi.org/10.1016/j.thromres.2020.04.013.

4. Panigada M, Bottino N, Tagliabue P, et al. Hypercoagulability of COVID-19 Patients in Intensive Care Unit. A Report of Thromboelastography Findings and Other Parameters of Hemostasis. J Thromb Haemost 2020 Apr 17 [ahead of print]; https://doi.org/10.1111/jth.14850.

5. American Society of Hematology. COVID-19 and VTE/Anticoagulation: Frequently Asked Questions. 5/18/2020. Available at https://www.hematology.org/covid-19/covid-19-and-vte-anticoagulation. Accessed May 30, 2020.

6. Helms J, Tacquard C, Severac F, et al. High risk of thrombosis in patients in severe SARS-CoV-2 infection: a multicenter prospective cohort study. Intensive Care Med. 2020 May 4 [ahead of print]; https://doi.org/10.1007/s00134-020-06062-x

7. Ullah W, Saeed R, Sarwar U, et al. COVID-19 Complicated by Acute Pulmonary Embolism and Right-Sided Heart Failure. J Am Coll Cardiol Case Rep 2020 Apr 17 [E-pub ahead of print]; https://doi.org/10.1016/j.jaccas.2020.04.008.

8. Varga Z, Flammer AJ, Steiger P, et al. Endothelial Cell Infection and Endotheliitis in COVID-19. Lancet 2020;395:1417-1418. doi: 10.1016/S0140-6736(20)30937-5.

9. European Working Group on Echocardiography. The European Cooperative Study on the clinical significance of right heart thrombi. Eur Heart J.1989;10:1046-1059.

10. National Institutes of Health. Antithrombotic Therapy in Patients with COVID-19. May 12, 2020. Available at: https://www.covid19treatmentguidelines.nih.gov/antithrombotic-therapy/. Accessed May 30, 2020. 

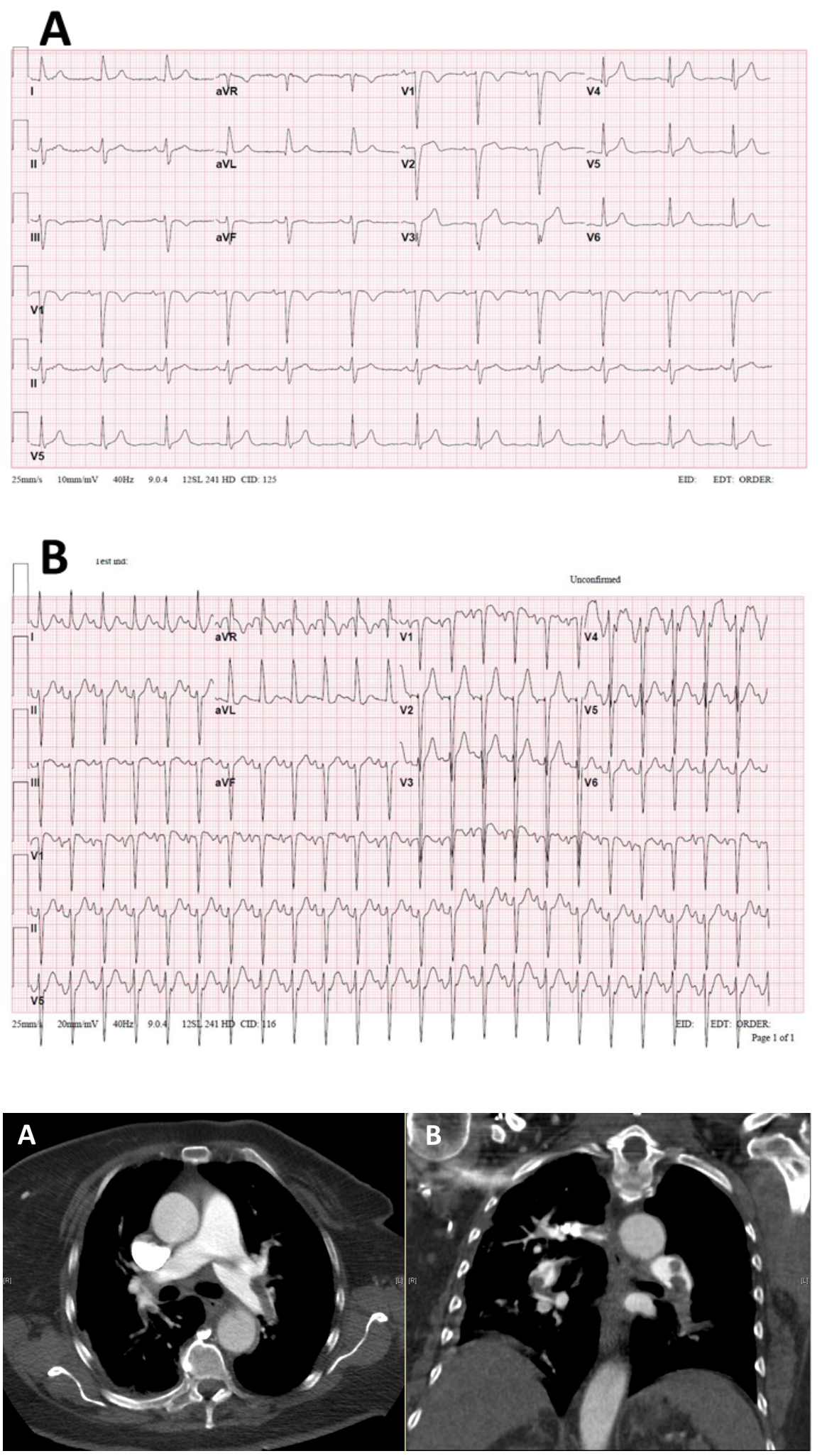


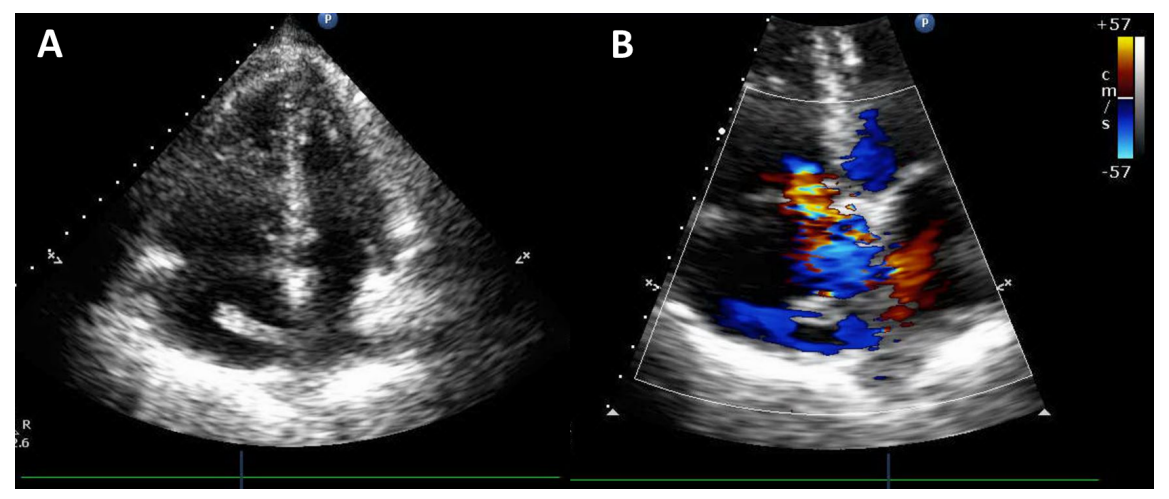

